\title{
History and General Characteristics of the Adaptive Control System of the Deuterium Pre-concentration Stage in Heavy Water Production
}

\author{
Karachenets Dmitryi \\ St. Derevlyanskaya, 19a, of.54, Kyiv 04119, Ukraine
}

\begin{abstract}
The history of creation in 1971-1975 years at SDB MMS of the Institute of Cybernetics (Kyiv city) the control system for the deuterium pre-concentration stage in heavy water production at Dneprodzerzhinsk chemical plant (now Kamianske city, PJSC "DneprAzot") is considered. It is given a technological block-diagram of the stage with its components and block-diagram of ACSTP which was developed in consequence of investigations of this complex managed object. A mathematical model of the distillation columns in the separating units of hydrogen isotopes which was used for synthesis of an algorithm of the adaptive stochastic optimal system with active accumulation of information about managed probabilistic processes is described. The results of the carried out tests of the system confirmed its efficiency.
\end{abstract}

Key words: Adaptive control system, deuterium, heavy water, distillation, ACSTP, mathematical model, managed probabilistic process.

\section{Introduction}

At the beginning of the theory of adaptive control systems when it comes to control under conditions of uncertainty, many experts in the field of control associate with the works of stochastic optimal control by A. A. Feldbaum. His concept of active accumulation of information in control systems with probabilistic interpretation of the tasks from the standpoint of the Bayesian approach, called the dual control [1], has found many followers, including in the Kyiv Institute of Cybernetics (IC) of the National Academy of Sciences of Ukraine (NASU). This approach has great difficulties in the implementation of the synthesized algorithms of control. However, under certain assumptions, which were not contrary to the real problem, it was able to create a highly effective adaptive control systems even with the limitations of

Corresponding author: Karachenets Dmitryi, Ph.D., research fields: mathematical, algorithmic, applied software and information support of automated control systems for technological processes, complexes, systems in the chemical industry and oil pipeline transport. the first Soviet control computing machines (CCM). One such system is the subject of this article.

\section{Experimental Section}

\subsection{Brief Information about Deuterium and Heavy} Water

The discovery of deuterium by the American physicists (the end of 1931, Harold Urey with followers, winner of the Nobel prize in chemistry in 1934) and heavy water (1933, Gilbert Lewis and Ronald McDonald) is on a par with the outstanding discoveries in 1932, the year of miracles or year anno mirabilis, as it was called by physicists (with the discovery of the neutron and positron and the creation of the proton-neutron theory of the structure of nuclei, the implementation of the first nuclear fusion reactions, etc.) [2]. These discoveries have radically changed over the last 85 years of life on earth, as also opened to humanity still not fully realized possibilities for the development of virtually inexhaustible energy sources.

Heavy water has found many applications. One of 
the main is the use of heavy water in nuclear reactors as a neutron moderator and coolant. To fill such a reactor it is necessary up to 100-300 tons of heavy water. They believe that heavy water and deuterium will play a big role in the industrial thermonuclear fusion in the future.

With today's technologies for the production of heavy water (an isotope exchange, electrolysis and burning of deuterium-enriched hydrogen) the cost on the world market is about $\$ 100-200$ per $1 \mathrm{~kg}$. Nevertheless, its production annually reaches several thousand tons.

Note that until 1946, the only used method of enrichment of natural waters was its electrolysis, in which the molecules of "light" water decomposed into oxygen and hydrogen-protium lighter than a molecule of heavy water into oxygen and hydrogen-deuterium. As a result of this process in electrolytic baths there is accumulated heavy water or, in other words, there is an increase in its concentration. The electrolysis process is very energy intensive. Therefore, in modern factories for first-stage concentration of deuterium, two-temperature counter-current hydrogen sulfide technology of isotope exchange is applied with the output concentration of heavy water 5-10\%. And in the second stage there is applied electrolysis of a solution of alkali at a temperature of about $0{ }^{\circ} \mathrm{C}$ with an output concentration of heavy water $99.75-99.995 \%$.

After 1946, industrial application was also received quite energy-consuming technology based on the rectification of liquid hydrogen [3]. One of the productions using this technology was put into operation in October 1965 at DDCP - Dneprodzerzhinsk chemical plant (now PJSC "DneprAzot" and Dneprodzerzhinsk now renamed Kamenskoe). The considered system was created for energy efficiency increasing of this production.

\subsection{General Description of Heavy Water Production at DDCP}

The production was secret. Open title of it was a "Gas Post-Treatment Production (GPTP)".
A block-diagram of the GPTP is shown in Fig. 1. The production consisted of two workshops ("A" and "B") which had got length of 300 meters, width of 50 meters and a height in a multistory building. Nitrogen $\left(\mathrm{N}_{2}\right)$-hydrogen $\left(\mathrm{H}_{2}\right)$ mixture $(\mathrm{NHM})$, which was intended for ammonia synthesis, served on the DDCP as input raw material for GPTP. (Why it was so complicated is unknown for the author).

Shop "B" of GPTP - technological — contained three stages of separation of hydrogen isotopes and installation for burning of deuterium and obtaining heavy water which was sending to the store for finished output. The flow of NHM passed to the catalytic purification from possible oxygen $\left(\mathrm{O}_{2}\right)$ impurities in a special installation before entering the shop "B".

The second and third stages of separation represented the installations, consuming a relatively small amount of energy. The first level as the level of deuterium pre-concentration was the main consumer of electricity coming into production. This stage (Fig. 2) consisted of 10 separating units (aggregates A1, ..., A10), each of which contained about 50 devices with different technological processes (reactors of catalytic purification of the mixture from impurities of oxygen, many different types of heat exchangers, nitrogen adsorbers-desorbers, expanders, turbo-expander on the stream of shared hydrogen and, as "heart", distillation column with 80 perforated plates).

Separating units were serviced by four refrigeration cycles: "recycled water" with a huge wooden hail towers, liquid ammonia $\left(\mathrm{NH}_{3}\right)$ as on the ice stadium, liquid nitrogen $\left(\mathrm{N}_{2}\right)$ and refrigerating hydrogen $\left(\mathrm{H}_{2}\right)$. And all technological devices in each separating unit were divided into three blocks: SB-"steel" (where flow temperatures had dropped from a high of around 2-3 hundred degrees by Celsius to the boiling point of liquid ammonia (approximately $-40{ }^{\circ} \mathrm{C}$ )), WB-“warm” (?!) (temperature range from $-40{ }^{\circ} \mathrm{C}$ to the boiling point of liquid nitrogen (about $-193{ }^{\circ} \mathrm{C}$ )) and CB-“cold" (temperature range from $-193{ }^{\circ} \mathrm{C}$ to the 

Stage in Heavy Water Production

boiling temperature of liquid hydrogen (about materials: ordinary cold-rolled steel in the "steel" $\left.-252{ }^{\circ} \mathrm{C}\right)$ ). According to these temperature ranges the block to copper in the "cold" block.

technological equipment was made of different

(GPTP -the gas post-treatment Production)

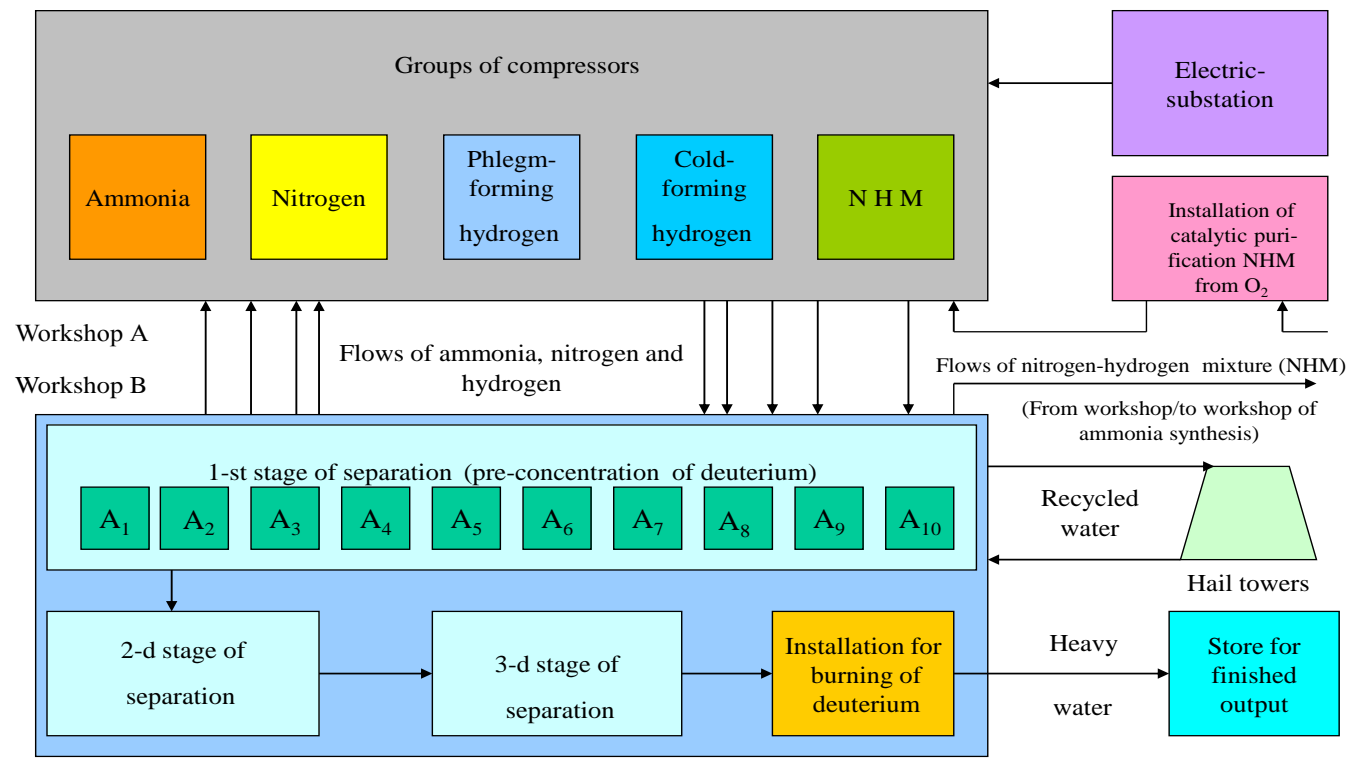

Fig. 1 A block-diagram of the GPTP.

of the stage of deuterium pre-concentration



Fig. 2 A block-diagram of the 1-st stage of separation of hydrogen isotopes (stage of deuterium pre-concentration). 


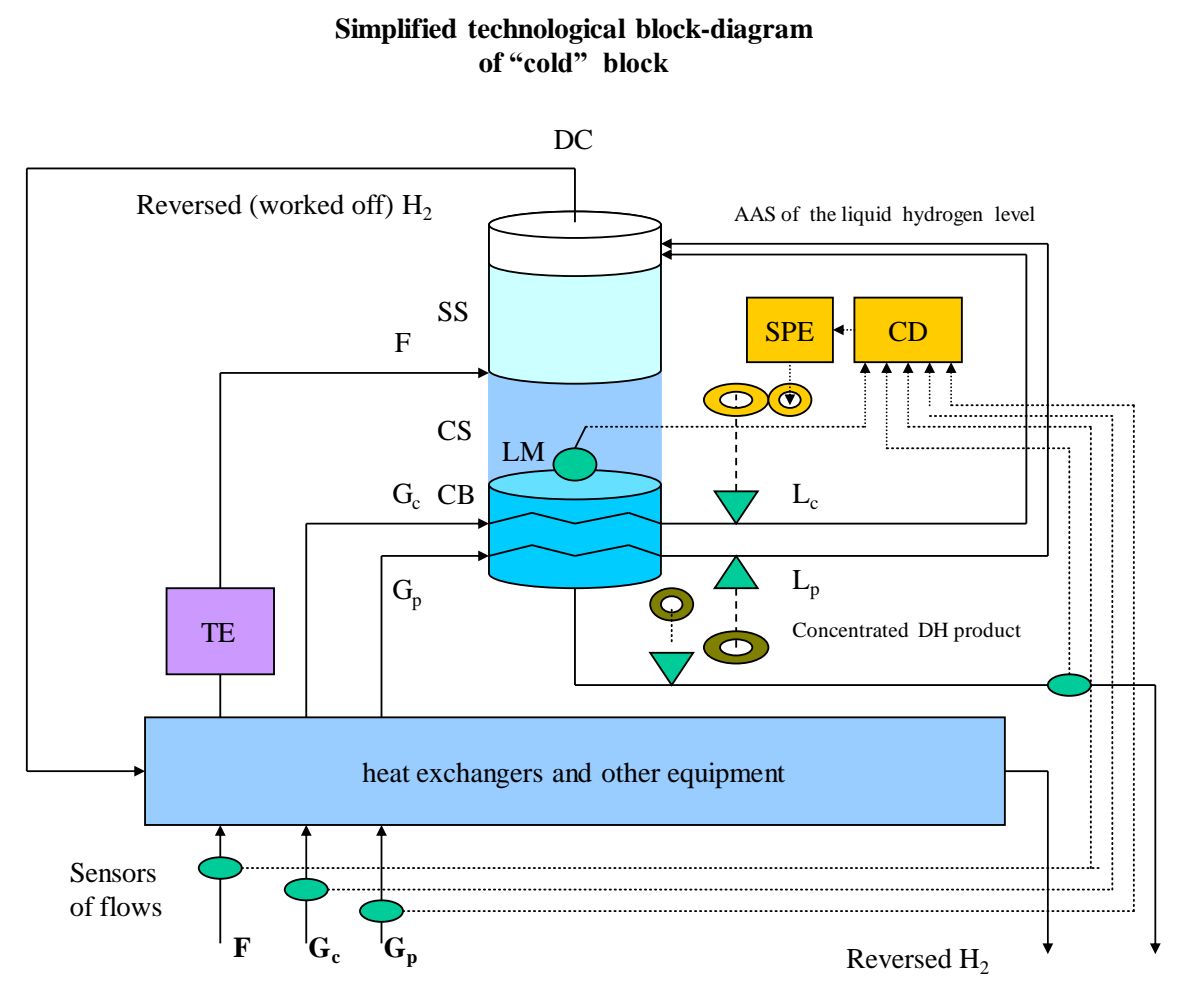

Fig. 3 The block-diagram of the "cold" block.

Maintaining a balance of "cold" in "cold" block (Fig. 3) of each the separation unit was achieved by regulating the level of liquid hydrogen in the cube (CB) of the distillation column (DC). This was done by changing to the column the cool forming hydrogen flow of $G_{c}=G_{c}$, which, after passing through a series of heat exchangers in three blocks $(\mathrm{SB}, \mathrm{WB}, \mathrm{CB})$ with cooling of the reverse flow of the exhaust stream of hydrogen, was passed through the coil pipes of the column cube as the last stage of cooling.

After throttling through the needle valve with a pressure of 200 atmospheres to about 1.4 atmosphere, it turned to steam, but not to introduce the reader in delusion, say, to a gas-liquid stream containing about $95 \%$ of the liquid phase $\mathrm{L}_{\mathrm{c}}$.

Stream $G_{p}=G_{p}$ of phlegmforming hydrogen entered into the column at approximately the same scheme, giving only $63 \%$ of the liquid phase $\mathrm{L}_{\mathrm{p}}$ as it was throttling only with pressure of about 3-4 atmospheres; but the flow of this stream was many times greater than the previous flow.

In Fig. 3 F, $G_{c}$ and $G_{p}$ values of the measurments at the input of CB for flows $F, G_{c}$ and $G_{p}$, come in DC. If $F_{u}, G_{u, c}$ and $G_{u, p}$ were values of the corresponding flows at the entrance to the separation unit $\mathrm{A}_{i}, i=1, \ldots$, 10 , then $\mathrm{G}_{\mathrm{c}}=\mathrm{G}_{\mathrm{u}, \mathrm{c}}, \mathrm{G}_{\mathrm{p}}=\mathrm{G}_{\mathrm{u}, \mathrm{p}}$ and

$$
\mathrm{F}=\beta \mathrm{H}, \mathrm{f} * \mathrm{Fu}
$$

where $\beta_{\mathrm{H}, \mathrm{f}}$-fractional hydrogen content in NHM.

Depleted by deuterium hydrogen flows from the tops of distillation columns of all 10 separation units were received in the common collector, were pressed in the cold- and phlegmforming hydrogen compressors with limited performance, and then re-distributed among all separation units. This led to a consideration of the entire deuterium pre-concentration stage as a united technological complex.

The 1st stage of hydrogen isotopes separation made it possible to vary the concentration of deuterium from natural $\sim 0.0156 \%$ (or $15.6 \gamma$, where $\gamma=10^{-5}$ ) to $4.5 \%$ (by volume). This concentration was determined by deuterohydrogen $\mathrm{DH}$ and accounted for 9\% (by volume) because D2 molecules of pure deuterium at low its concentrations in the general flow of hydrogen 
are almost non-existent: deuterium in molecules is "diluted" by protium $\mathrm{H}$.

Shop "A" of GPTP (Fig. 1) contained about 20 powerful compressors with motor drive, having consumed a day about 1.2 million kilowatt-hours of electricity from a special electricsubstation that was installed next to the workshop "A". Compressors provided compression to the units, tens and hundreds of atmospheres streams of divided gas (NHM), gaseous ammonia, nitrogen, phlegm- and coldforming hydrogen.

\subsection{Brief Characteristics of the Process Control System of GPTP}

According to the GPTP draft developed in the Soviet Union by GIAP — a leading (main) research Institute of nitrogen industry (MINI), Moscow, content (by volume) of deuterium atoms in the waste (depleted with deuterium) stream of hydrogen leaving the deuterium pre-concentration stage was in the range of $1.4 \gamma$ (or $1.4 \times 10^{-5}$ ). In fact, this content over 4 years of work GPTP (early 1970s) was managed to reduce only to $2.3 \gamma$, which corresponded to 93.7 per cent from the project $(100 \%)$ degree of extraction.

To solve the problem of increasing the degree of deuterium extraction to the project one tried employees of GPTP, and Central GIAP, and Dneprodzerzhinsk branch of the GIAP, and created on DDCP special research laboratory at GPTP, and ... Where not only were they looking for a "dog"? May it be an error in the project? May it be something not so installed? May it be (there was even a such version!) physicists have incorrectly defined the properties for the volatility of hydrogen isotopes in nonideal mixture of protium and deuterium?

On the production something has regularly been improved. Each year, the staff of GPTP assumed the obligation to increase output of heavy water by $0.1 \%$.

One of the ways to solve this problem, the leadership of GPTP saw as to create a more perfect system of production management and, above all, in building a process control system as the Automated Control System of Technological Processes (ACSTP).

On GPTP in 1970 there was acquired CCM CM-1 with volume 4 Kilowords (8 Kilobytes) of RAM (main memory) and 6 Kilowords (12 Kilobytes) of read only memory, ROM (permanent storage devices) and by workers of GPTP service of Instrumentation and automation began the creation of a subsystem of gathering operational information (from the sensors of flow rate, pressure and temperature) with hourly printing data on a special paper tape (Fig. 4). Further, this subsystem will be determined as a subsystem 1 of the ACSTP. However, it could not solve problem.

This was achieved by creating in the composition of the ACSTP an adaptive control system (ACS), optimizing the deuterium pre-concentration stage. This system was created by a small team of employees of the Department "Mathematical and Algorithmic Maintenance of the ACSTP" in Special Design Bureau of Mathematical Machines and Systems (SDB MMS) IC NASU under the direction of the author of this article.

\subsection{Pre-project Investigation of GPTP}

Performed research works on the synthesis of ACS included:

(1) analysis of GPTP as a whole as the managed or controled object (CO);

(2) a similar analysis of the deuterium pre-concentration stage (1st stage of separation of hydrogen isotopes);

(3) development of mathematical models of technological processes on GPTP;

(4) study of mathematical models of the separation process of hydrogen isotopes in the DC for the separation units of the 1-th stage;

(5) synthesis of the ACS;

(6) development of automatic adjusting system (AAS) of the level of liquid hydrogen in a cube of DC.

Analysis on the items 1 and 2 showed that the task 


\section{History and General Characteristics of the Adaptive Control System of the Deuterium Pre-concentration Stage in Heavy Water Production}

of optimization of technological equipment of GPTP at a selectable maximum permissible NHM loads of the separation units A1, ..., A10 (Fig. 1) should be addressed in two directions: reduction of energy consumption for processing NHM and increase of the degree of extraction from it of deuterium. So, to keep the restrictions on the enthalpy flows into a "cold" blocks of separation units, by the first criterion (lower energy) there could be considered management tasks of the refrigeration cycles in these units, and with them the management optimization of compressor equipments of workshop "A".

The solution of all problems was made possible by mathematical modeling as the process of fractionation of hydrogen isotopes, and complex chemical-technological schemes of each separation unit and the entire 1st stage of hydrogen isotopes separation (the items 3 and 4).

The results of the performed studies on energy savings in GPTP have been a number of proposals to the leadership of GPTP for the improvement of the rules of operation of technological equipment of the workshops of "A" and "B".

The main effort of the team was aimed at creating ACS, which was implemented in two subsystems of GPTP ACSTP (Fig. 4):

- subsystem 2 for identification of mathematical models of DC (in the separation units);

- subsystem 3 for calculations, using the identified mathematical models of DC, of optimal controls $U_{i}, i=$ $1, \ldots, 10$, as phlegmforming flows of hydrogen supplied to DC, with a total limited resource.

Fragments of the program worked out beforehand in main memory of CCM CM-1 and subsequently "sutured" into the ROM. This ensured high reliability of the entire system.

Because in the course of the optimization system working it was assumed a regular (minimum once per shift) adjustment of phlegm, supplied in the separation

Block-dingram of GPTP ACSTP with the adap tiv e control system (ACS)

for the deuterium pre-concentration stage

Subsystens 2 and 3 \& ACS- the progran complexesfor COMI CMF-1

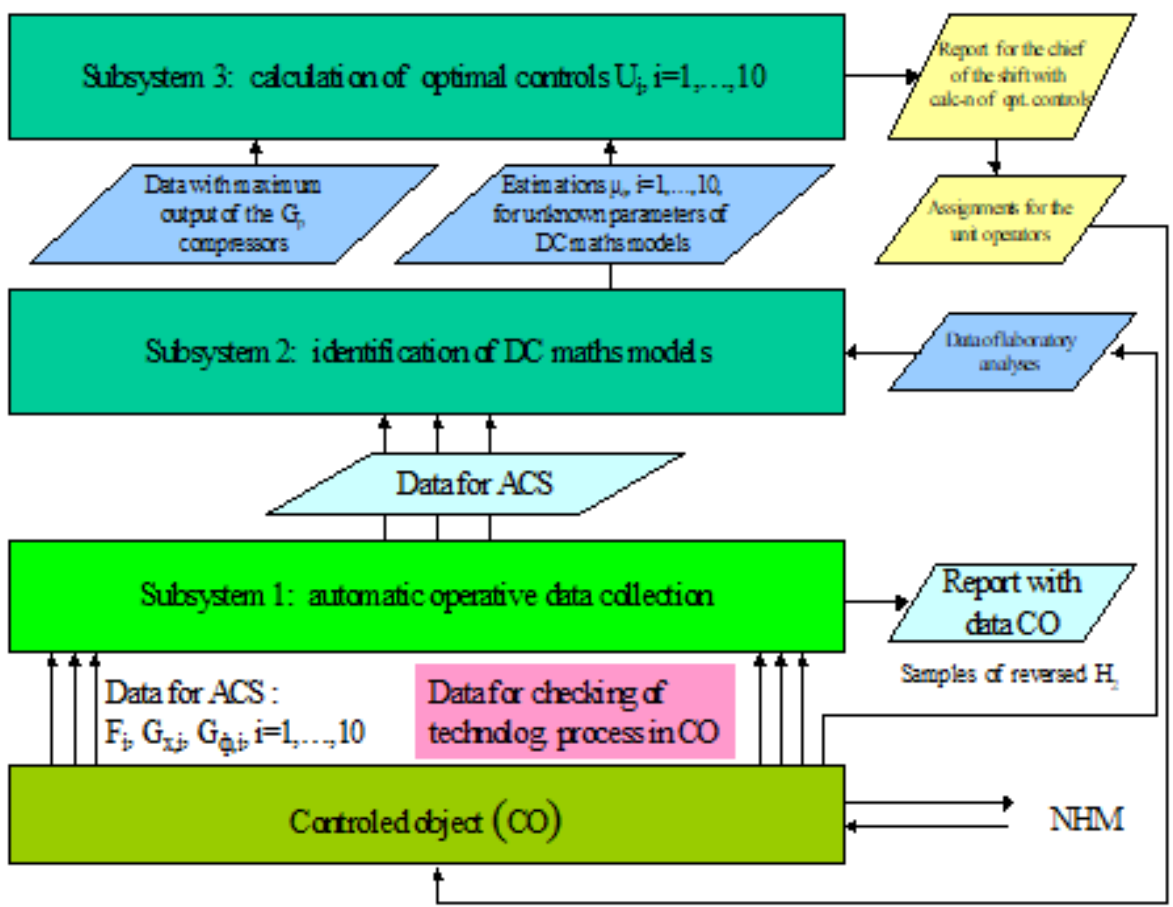


Fig. 4 Block-diagram of GPTP ACSTP with the adaptive control system for the deuterium pre-concentration stage.

units, it was necessary to take care of the creation of above mentioned AAS, which would free unit operators from manual adjustment.

Level adjustment by means of the needle valve was carried out by the operators through a long (probably 3 meters) stock. In AAS the actuator for stock was SPE-“step" pneumatic engine (Fig. 3). The choice of SPE as a driver was determined by the fact that GPTP was a production with a high level of fire and explosion. Pneumatic control signal to "step" engine was produced in the control device $(\mathrm{CD}$, Fig. 3) of AAS according to the special algorithm on the basis of several sensors indications of the parameters values of a "cold" block: level of liquid hydrogen in a cube of DC generated by the sensor LM (the level measurer); sensors of input (for CB) flows $F, G_{c}, G_{p}$ and an output flow- "concentrated DH product" in Fig. 3.

Here, $\mathrm{F}$ is the hydrogen flow that was allocated in the WB from the flow of NHM, which after passing through the heat exchange equipment in $\mathrm{CB}$ finally cooled to dew point in the turbine expander (TE). Then it was driven up on a "food" plate of DC (food by a stream of separated hydrogen), which divided distillation column to the comprehensive (CS) and strengthening (SS) sections (Fig. 3).

This AAS was protected by the copyright certificate [4].

\subsection{By the Use of Mathematical Models in a Control System of GPTP}

GPTP investigations carried out led the author of the article, who was directing (both scientific and organizational) on the development of optimal control system for GPTP, to the conclusion that the misunderstandings associated with insufficient degree of deuterium extraction and, consequently, the shortfall in production of commercial product (heavy water), were caused primarily by errors of the sensors of the gas flows (Fig. 3) in a deep cold (at the temperature of $-193{ }^{\circ} \mathrm{C}$ ) and by the imperfection of control for the 1st separation stage. The process of rectification, as experts know in this area is very sensitive in a phlegm number or reflux ratio (the ratio of the phlegm flow which is returned from the top of the column on its irrigation, with separated flow of the mixture coming from the outside to the column). The dependence of deuterium concentration in exhaust (worked off) gas, as expected [5], submitted an almost exponential character in the workspace. The increase in reflux ratio has reduced, but not enough, the deuterium content in the exhaust. Reducing reflux ratio by the same amount resulted in significantly greater increase in the content of deuterium in it.

In addition, the degree of isotope separation in the column for all stable values of the flows entering the column was subjected to "drifting" as it was identified during the processing of a large statistical material which had been accumulated on the modes of one of the distillation columns. Translated into reflux ratio, changes of the last looked like a random process for which there fitted the model of the so-called in the theory of random processes, Markov process. "Drifting" reflux ratio of each separation unit DC was caused by several factors: vibration of DC because of compressor equipment which worked in the workshop "A", common to all units "drifting" share $\beta_{\mathrm{H}, \mathrm{f}}(1)$ hydrogen in NHM flow, and changes in the heat content of the gas flows $F, G_{c}$ and $G_{p}$ (Fig. 3) which arrived in the $\mathrm{DC}$ and depended on the equipment operation modes of all blocks (SB,WB and CB) of the particular unit.

Control technology adopted earlier on the GPTP, was reduced to a simple algorithm: equally, usually between all separation units were distributed received on the GPTP flow of nitrogen-hydrogen mixture and evenly - the flow of phlegm, the resource of which was limited by the performance of the two phlegmforming hydrogen compressors. It is clear that all distributions were made by the actual readings of 
flow meters of NHM gas and phlegmforming hydrogen. However, due to measurement errors the actual reflux ratio for different columns differed from each other in a random way that for the whole deuterium pre-concentration stage it resulted in a decrease in the degree of extraction.

Solving this problem was made possible by establishing, appropriate to the task and the real possibilities for its use, a mathematical model of the distillation columns of the separation units. This model was built in two stages.

First, on the basis of physical laws, it has carried out a detailed mathematical description of the processes occurring in DC and associated process equipment (created, say, a "complete" model), developed a program for the calculation of such processes (this model) on a digital computer (primarily on the BECM-6, which was installed and operated in IC; the programming language was FORTRAN), conducted an experimental study of the modes of the columns of the separation units in GPTP (mainly on the DC of unit A3, Fig. 1, and optionally at DC of other units), experimental data were treated and parameters of the "full" model of DC and disturbances were refined.

In the second stage, by calculations of DC modes on its "full" model the original data were received to determine the parameters of selected "simplified" model. We considered various versions for such a model.

The most "simplified" was the model:

$$
\mathrm{y}_{\mathrm{i}, \mathrm{s}}=\alpha_{\mathrm{y}} \exp \left(0.5 \mu_{\mathrm{i}, \mathrm{s}}+\mathrm{U}_{\mathrm{i}, \mathrm{s}}\right)
$$

where:

$\mathrm{y}_{\mathrm{i}, \mathrm{s}}$ - the deuterium concentration in the gas stream of depleted, worked off hydrogen from the top of the $\mathrm{i}$-th $\mathrm{DC}, \mathrm{i}=1, \ldots, 10$, was working in permanent, main mode of operation of the entire 1st separation stage, in the s-th cycle;

$\alpha_{y}-\mathrm{a}$ constant, which is equal $1.4 \times 10^{-5}$ or $1.4 \gamma$, the same coefficient for all DC;

$\mu_{\mathrm{i}, \mathrm{s}}$ - uncontrollable random disturbance affecting the separation process of hydrogen isotopes in the i-th DC in s-th cycle (for different $i, j$ values $\mu_{\mathrm{i}, \mathrm{s}}$ and $\mu_{\mathrm{j}, \mathrm{s}}$ were taken to be independent);

$\mathrm{U}_{\mathrm{i}, \mathrm{s}}$ - control (control effect) on the separation process of hydrogen isotopes in the i-th DC in s-th cycle associated with the consumption of phlegmforming hydrogen $\mathrm{G}_{\mathrm{p}, \mathrm{i}, \mathrm{s}}$ applied to the i-th DC in s-th cycle by correlation:

$$
\mathrm{G}_{\mathrm{p}, \mathrm{i}, \mathrm{s}}=\mathrm{G}_{\mathrm{p}, \mathrm{b}}+\eta_{\mathrm{i}, \mathrm{s}} \mathrm{U}_{\mathrm{i}, \mathrm{s}} \cdot
$$

In Eq. (3) $G_{p, b}-a$ some basic value for phlegmforming hydrogen flow in DC, determined from the average of resource $G_{p, s}\left(G_{p}\right.$, Fig. 4) on one unit for all operating units, and $\eta_{\mathrm{i}, \mathrm{s}}$ is the scaling factor, the values of which were calculated taking into account the ratio of fluid flow received DC on its top plate with the flows $G_{p}$ and $G_{c}$, and gas flow $F$ which came at the dew point on a food plate of DC. The value of stream $G_{c}$ was determined by calculation from the expression:

$$
\mathrm{G}_{\mathrm{c}, \mathrm{i}, \mathrm{s}}=\beta_{\mathrm{i}, \mathrm{s}}+\beta_{\mathrm{i}, \mathrm{s}}^{\mathrm{f}} \mathrm{F}_{\mathrm{i}, \mathrm{s}}+\beta_{\mathrm{i}, \mathrm{s}}^{\mathrm{p}} \mathrm{G}_{\mathrm{p}, \mathrm{i}, \mathrm{s} 。}
$$

The coefficients $\beta_{\mathrm{i}, \mathrm{s}}, \beta_{\mathrm{i}, \mathrm{s}}^{\mathrm{f}}, \beta_{\mathrm{i}, \mathrm{s}}^{\mathrm{p}}$ in Eq. (4) were clarified by programs of the ACSTP subsystem 2 (identification of DC mathematical models) according to the measurements of subsystem 1 (automatic operative data collection) with the use of recurrent least squares method.

In Eq. (2) $\mathrm{y}_{\mathrm{i}, \mathrm{s}}=1.4 \gamma$ if $\mu_{\mathrm{i}, \mathrm{s}}=0, \mathrm{U}_{\mathrm{i}, \mathrm{s}}=0$, which corresponds to the value that was admitted in the project of GPTP developed by GIAP.

Below for example, the expression (2) is given by selective discrete values in a table and by graphs in Fig. 5.

For describing of the changing of process $\mu_{\mathrm{i}, \mathrm{s}}$ it used the model of a stationary discrete-continuous Markov process with transition probability density defined by the expression (5)

$$
p\left(\mu_{i, s} / \mu_{i, s-1}\right)=\frac{1}{\sigma_{\mu} \sqrt{2 \pi}} \exp \left\{-\frac{\left(\mu_{i, s}-\alpha_{\mu} \mu_{i, s-1}\right)^{2}}{2 \sigma_{\mu}{ }^{2}}\right\},
$$

where $0<\alpha_{\mu}<1$.

In Eq. (5) for the parameters $\alpha_{\mu}$ and $\sigma_{\mu}$, where $\sigma_{\mu}{ }^{2}$-dispersion, the index " $\mathrm{i}$ " is omitted, because their 
values after sampling tests were adopted the same for all units.

Table 1 Dependence of the concentration values $y_{i, s}$ in $\gamma\left(=10^{-5}\right)$ from dimensionless disturbance $\mu_{i, s}$ and control $U_{i, s}$ values.

\begin{tabular}{llllllllll}
\hline$\mu_{\mathrm{i}, \mathrm{i} \backslash} \mathrm{U}_{\mathrm{i}, \mathrm{s}}$ & $-3,0$ & $-2,5$ & $-2,0$ & $-1,5$ & $-1,0$ & $-0,5$ & 0 & 0,5 & 1,0 \\
\hline-2 & - & - & - & - & 10,36 & 6,26 & 3,81 & 2,30 & 1,40 \\
-1 & - & - & - & 10,36 & 6,26 & 3,81 & 2,30 & 1,40 & 0,85 \\
0 & - & - & 10,36 & 6,26 & 3,81 & 2,30 & 1,40 & 0,85 & 0,52 \\
1 & - & 10,36 & 6,26 & 3,81 & 2,30 & 1,40 & 0,85 & 0,52 & 0,31 \\
2 & 10,36 & 6,26 & 3,81 & 2,30 & 1,40 & 0,85 & 0,52 & 0,31 & 0,19 \\
\hline
\end{tabular}

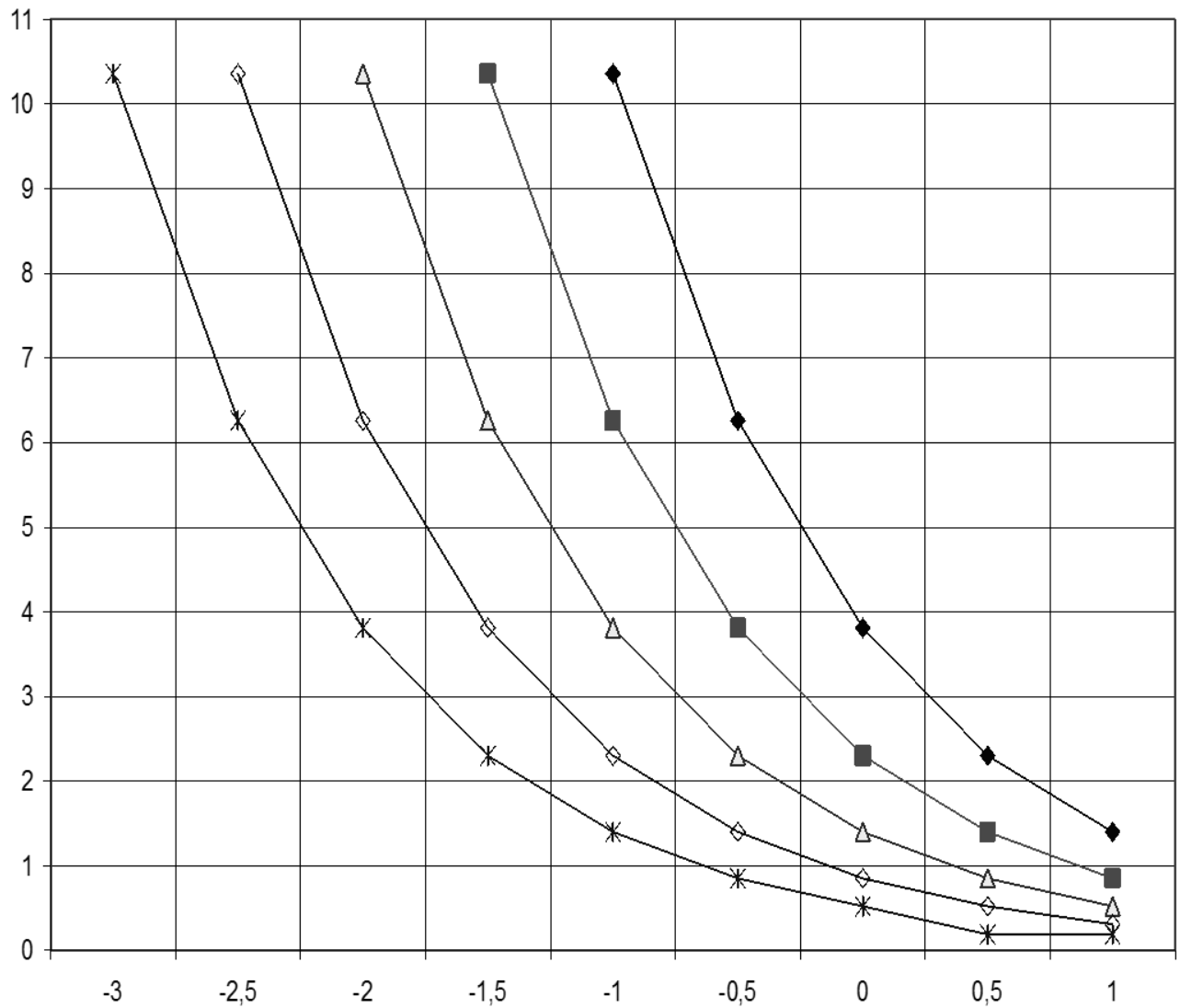

Fig. 5 Graphs of the concentration values $y_{i, s}$ in $\gamma$ from the dimensionless values of control $U_{i, s}$ with different dimensionless values of disturbance $\mu_{\mathrm{i}, \mathrm{s}}: \mu_{1}=-2 ; \mu_{2}=-1 ; \mu_{3}=0 ; \mu_{4}=1 ; \mu_{5}=2$.

\subsection{The Description of the General Algorithm of} Functioning of the Adaptive Control System and Its Subsystem of Identification of DC Mathematical Models

Every time beat $s$ in Eq. (2) to Eq. (5) corresponded to the same shift of work on the GPTP, which lasted 8 hours. By the beginning of each shift the operator of CCM (OCCM) has been obtained from the laboratory test results of gas streams from the top of DC units. The samples of these streams were selected at the beginning of the previous (s-1) shift and the analysis of them lasted for several hours (about 6 hours). OCCM carried out of them processing using software subsystem 2 of ACSTP (Fig. 4).

Ordinarily, the analyses in each shift were not carried out for all units, but during the day (three shifts) each unit was subjected to such analysis at least once. As to the deuterium content in the overall flow worked off hydrogen, each shift was determined, although it was not used in the calculations, and served only to 
control the course of technological processes throughout the whole deuterium pre-concentration stage.

In the beginning of the shift its chief (SC), based on your own evaluation of state of units and the planned tasks for the production, pointed OCCM loads $\mathrm{F}_{\mathrm{i}, \mathrm{s}}, i=1,2, \ldots, k$, where $k$ is the number of working units $(k \leq 10)$, for NHM stream of each unit in the s-th shift (Usually worked all 10 units). If necessary, the resource $G_{p, s} \quad\left(G_{p}\right.$, Fig. 4) of phlegmforming hydrogen was adjusted in CCM. Using the programs of subsystem 3 of ACSTP OCCM calculated optimal distribution of resource $G_{p, s}$ between units.

The SC is issued to the operator of each i-th unit $\left(\mathrm{OA}_{\mathrm{i}}\right)$ assignments for flows of $\mathrm{F}_{\mathrm{i}, \mathrm{s}}$ and $\mathrm{G}_{\mathrm{p}, \mathrm{i}, \mathrm{s}}\left(=\mathrm{G}_{\mathrm{p}, \mathrm{i}, \mathrm{s}}\right)$. After realization of the assignments $\mathrm{OA}_{\mathrm{i}}$ regulated, if necessary, the consumption of coldforming hydrogen $\mathrm{G}_{\mathrm{c}, \mathrm{i}, \mathrm{s}}$ till there was reaching a stable level of liquid hydrogen in the DC cube of i-th unit.

About reaching stable conditions at the $\mathrm{A}_{\mathrm{i}}$, either by manual or by automatic adjustment when AAS was installed on the i-th unit, $\mathrm{OA}_{\mathrm{i}}$ reported OCCM. Latest with the help of programs of the ACSTP subsystem 2 carried out the adjustment of the coefficients $\beta_{\mathrm{i}, \mathrm{s}}, \beta_{\mathrm{i}, \mathrm{s}}^{\mathrm{f}}$ and $\beta_{\mathrm{i}, \mathrm{s}}^{\mathrm{p}}$ included in the expression (4), for using them on the next cycle $(s+1)$. But in calculation of the optimal values $G_{p, i, s}, i=1, \ldots, k$, of phlegmforming hydrogen flow for DC of each unit, which was working, the values of coldforming hydrogen were determined according to Eq. (4) with the values of $\beta_{\mathrm{i}, \mathrm{s}-1}, \beta_{\mathrm{i}, \mathrm{s}-1}^{\mathrm{f}}$ and $\beta_{\mathrm{i}, \mathrm{s}-1}^{\mathrm{p}}$ for these coefficients that have been received to the s-th cycle.

Adaptive control system (composed of software subsystems 2 and 3 in the ACSTP, Fig. 4) was developed as optimal stochastic system, which was to provide the minimum of function of expected average deuterium losses in each upcoming shift $\mathrm{s}(\mathrm{s}=1,2, \ldots)$ in the form:

$$
\rho_{s}=\lim \frac{1}{n} \sum_{n=1,2, \ldots}^{s+n-1} R_{t}
$$

where specific risk:

$$
\mathrm{R}_{\mathrm{t}}=\mathrm{M}\left\{\mathrm{y}_{\mathrm{t}}\right\} .
$$

Here $\mathrm{M}$ is the symbol of mathematical expectation of random variables $\mathrm{y}_{\mathrm{t}}, \mathrm{t}=\mathrm{s},(\mathrm{s}+1), \ldots$.

In turn, the concentration of deuterium $\mathrm{y}_{\mathrm{s}}$ in exhaust (worked off) gas, served as an effectiveness indicator of the technological processes of the deuterium pre-concentration stage on the s-th time, was defined as:

$$
y_{s}=\sum_{i=1}^{k} V_{i, s} y_{i, s} / \sum_{i=1}^{k} V_{i, s},
$$

where $\mathrm{V}_{\mathrm{i}, \mathrm{s}}$ is the total hydrogen flow, depleted in deuterium and leaving the i-th DC on the s-th time, which, excluding very small flow of concentrate from the cube $\mathrm{DC}$, was defined as:

$$
\mathrm{V}_{\mathrm{i}, \mathrm{s}}=\mathrm{F}_{\mathrm{i}, \mathrm{s}}+\left(\mathrm{G}_{\mathrm{p}, \mathrm{i}, \mathrm{s}}-\mathrm{L}_{\mathrm{p}, \mathrm{i}, \mathrm{s}}\right)+\left(\mathrm{G}_{\mathrm{c}, \mathrm{i}, \mathrm{s}}-\mathrm{L}_{\mathrm{c}, \mathrm{i}, \mathrm{s}}\right) .
$$

Recall that in Eq. (9):

$$
\mathrm{L}_{\mathrm{p}, \mathrm{i}, \mathrm{s}}=0,63 \mathrm{G}_{\mathrm{p}, \mathrm{i}, \mathrm{s}} ; \mathrm{L}_{\mathrm{c}, \mathrm{i}, \mathrm{s}}=0,95 \mathrm{G}_{\mathrm{c}, \mathrm{i}, \mathrm{s}} .
$$

Values $\mathrm{y}_{\mathrm{i}, \mathrm{s}}, \mathrm{i}=1,2, \ldots, k(k \leq 10)$ in $(8)$, determining the concentration of deuterium in the flow of $\mathrm{V}_{\mathrm{i}, \mathrm{s}}$ and exposed, according to Eq. (2), random perturbations $\mu_{\mathrm{i}, \mathrm{s}}$, were random variables.

Control variables of $U_{i, s}$ at each step s of the system operation had to be chosen so as to minimize the value of the functional $\rho_{\mathrm{s}}(6)$ and answer to constraints on resource of phlegmforming hydrogen:

$$
\sum_{i=1}^{k} \mathrm{G}_{\mathrm{p}, \mathrm{i}, \mathrm{s}} \leq \mathrm{G}_{\mathrm{p}, \mathrm{s}}
$$

In the absence of observations of perturbations $\mu_{\mathrm{i}, \mathrm{s}}$, $\mathrm{i}=1,2, \ldots, k(k \leq 10)$, representing the Markov (conditional) processes (5), they must have been perceived by a casual observer (in this case of the considered ACS) as a random (independent) values, the probability distribution (probability density) was marginal (unconditional) distribution laws generated by the processes (5). Such laws were normal (Gauss) laws whose mathematical expectation (M.E.) and standard deviation (M.S.D.) is 
equal to:

$$
\mathrm{m}_{\mu \infty}=0 ; \quad \mu \infty=\frac{\sigma_{\mu}}{\sqrt{1-\alpha_{\mu}{ }^{2}}} .
$$

Thus, before running in subsystem 2 as part of ACSTP (identification subsystem of DC models in ACS), priori information about the uncontrolled perturbations $\mu_{\mathrm{i}, \mathrm{s}}, \mathrm{i}=1,2, \ldots, k(k \leq 10), \mathrm{s}=1$, of technological processes in DC could be represented by a fully normal distributions of these variables $\mathrm{N}\left(\mu_{\mathrm{i}, \mathrm{s}}\right.$ $\mathrm{m}_{\mathrm{i}, \mathrm{s}}, \mathrm{D}_{\mathrm{i}, \mathrm{s}}$ ) with two sufficient statistics $\mathrm{m}_{\mathrm{i}, \mathrm{s}}=\mathrm{m}_{\mathrm{i}, 1}$ and $D_{i, s}=D_{i, 1}$, where " $m$ " and "D" symbols of M.E. and variance (square of the M.S.D.):

$$
\begin{gathered}
\mathrm{m}_{\mathrm{i}, 1}=0, \mathrm{i}=1,2, \ldots, \mathrm{k}(\mathrm{k} \leq 10), \\
\mathrm{D}_{\mathrm{i}, 1}=\left(\sigma_{\mu \infty}\right)^{2} .
\end{gathered}
$$

After entering into the subsystem 2 of data about the test results of the variables $\mathrm{y}_{\mathrm{i}, 1}$ of deuterium content in the samples, which were taken away from gas streams leaving the top of $\mathrm{DC}_{\mathrm{i}}, \mathrm{i}=1,2, \ldots, k$, in the beginning of the time beat $\mathrm{s}=1$ (1st shift), it might be calculated a posteriori laws of probability distributions of perturbations $\mu_{\mathrm{i}, 1}$ taking into consideration additive measurement errors $h_{i, s}$ of deuterium content. The latter were subordinated to the normal law with zero mathematical expectation (M. E.) and M.S.D. $\sigma_{h}=0.2 \gamma$. For different $i$ and $j$ the errors of $h_{i, s}$ and $h_{j, s}$ were an independent random variables.

According to Eq. (2), a posteriori law of probability distributions of perturbations $\mu_{\mathrm{i}, 1}$ had to have a quite complicated form. Storage of knowledge about them would require significant resources of RAM CCM CM-1 in a control system of GPTP, which was very limited (only $8 \mathrm{~KB}$ ).

Therefore, the dependence (2) was approaching by means of the linearized characteristic in the area of virtually the most possible values of the random variables $\mu_{\mathrm{i}, \mathrm{s}}$, or in other words, in the region of a priori mathematical expectations $\mathrm{m}_{\mathrm{i}, \mathrm{s}}$, which for $\mathrm{s}=1$ was determined for all $\mathrm{i}$ as 0 (12). In this case the conditional distribution $p\left(\tilde{y}_{i, s} / \mu_{i, s}, \quad U_{i, s}\right)$ can be represented as a normal distribution $\mathrm{N}\left(\tilde{y}_{\mathrm{i}, \mathrm{s}} ; \widetilde{m} y_{\mathrm{i}, \mathrm{s}}, \widetilde{D} y_{\mathrm{i}, \mathrm{s}}\right)$ with mathematical expectation and variance which must be equal to:

$$
\begin{gathered}
\widetilde{m}_{i, s}^{y_{i}=} \frac{1}{\chi_{i, s}}\left(\frac{1}{B}-\mathrm{m}_{\mathrm{i}, \mathrm{s}}+\mathrm{U}_{\mathrm{i}, \mathrm{s}}\right), \\
\widetilde{D^{y}}{ }_{i, s}=\sigma_{\mathrm{h}}{ }^{2} .
\end{gathered}
$$

The value of $\chi_{i, s}$ in Eq. (14) was determined by the expression:

$$
\chi_{\mathrm{i}, \mathrm{s}}=\frac{1}{A B \exp \left(B m_{i, s}+\mathrm{CU}_{i, s}\right)} .
$$

Here the coefficients $\mathrm{A}, \mathrm{B}$ and $\mathrm{C}$ belonged to the "simplified" model more general than model (2) for which $\mathrm{A}=\alpha_{\mathrm{y}}, \mathrm{B}=0.5$ and $\mathrm{C}=1$.

For such a local linearization of model (2), the posterior distributions of the disturbances $\mu_{\mathrm{i}, \mathrm{s}}$, which were calculated by means of the Bayes formula, were a kind of normal (Gauss) laws with statisticians defined by the expressions:

$$
\begin{gathered}
\widetilde{m}_{i, s}=\frac{\mathrm{m}_{\mathrm{i}, \mathrm{s}}-\left(\sigma \chi_{\mathrm{h}}\right)_{\mathrm{i}, \mathrm{s}}{ }^{2}+\mathrm{y}^{\chi_{i, s} \mathrm{D}_{\mathrm{i}, \mathrm{s}}}}{\mathrm{D}_{\mathrm{i}, \mathrm{s}}+\left(\sigma \chi_{\mathrm{h}}\right)_{\mathrm{i}, \mathrm{s}}{ }^{2}} ; \\
\widetilde{D}_{i, s}=\frac{\mathrm{Di}, \mathrm{s}\left(\sigma \chi_{\mathrm{h}}\right)_{\mathrm{i}, \mathrm{s}}{ }^{2}}{\mathrm{Di}, \mathrm{s}+\left(\sigma \chi_{\mathrm{h}}\right)_{\mathrm{i}, \mathrm{s}}{ }^{2}},
\end{gathered}
$$

where

$$
\begin{gathered}
y \chi_{i, s}=\chi_{\mathrm{i}, \mathrm{s}}+\mathrm{m}_{\mathrm{i}, \mathrm{s}}-1 / \mathrm{B} \\
\left(\sigma \chi_{h}\right)_{i, s}{ }^{2}=\chi_{\mathrm{i}, \mathrm{s}}{ }^{2}+\sigma_{\mathrm{h}}{ }^{2} .
\end{gathered}
$$

Further a priori to the time $(\mathrm{s}+1)$ the distributions of the disturbances $\mu_{\mathrm{i}, \mathrm{s}}$, determined by taking into account (5), wore also the nature of the normal laws with statistics:

$$
\begin{gathered}
\mathrm{m}_{\mathrm{i}, s+1}=\alpha_{\mu} \widetilde{m}_{i, s} ; \\
\mathrm{D}_{\mathrm{i}, s+1}=\alpha_{\mu}{ }^{2} \widetilde{D}_{i, s}+\sigma_{\mu}{ }^{2} .
\end{gathered}
$$


If in the time beat $s=1$ for the DC of separating units $j 1, j 2, \ldots, j p(p \leq k)$ the gas samples from the top of the columns for analysis were not taken away, then the statistics of $m_{i, 1}$ and $D_{i, 1}$ of the a priori to the time beat $\mathrm{s}=1$ the distributions of the corresponding perturbations were taken as variables $\widetilde{m}_{i, 1}$ and $\widetilde{D}_{i, 1}$ in the expressions (21) and (22).

Further processing of the analyses of the variables $\mathrm{y}_{\mathrm{i}, \mathrm{s}}, \mathrm{i}=1,2, \ldots, k,(k \leq 10), \mathrm{s}=2,3, \ldots$, which determined the concentration of deuterium in the gas flows from the tops of the DC was lasted similarly.

In accordance with expressions (14-22) it is easy to see how controls which were taken in the ACS in step $\mathrm{s}$, affect the precision of disturbances determination on future steps. This reflects the dual nature of the controls $U_{i, s}$, chosen in each cycle of operation of the adaptive control system.

\subsection{Brief Description of the Subsystem of Optimal Control Calculations}

Comprehensive study of the synthesis problem of the control algorithm in the ACS was preceded software implementation of the ACSTP subsystem 3. The limited size of the article does not allow describing the obtained results. Briefly the operation of this subsystem can be described as follows.

It is known [1] that in the Bayesian tasks the controls, which should report a minimum of specific risk (7), were founded from minimization of the conditional specific risk $r_{t}$. In this task it is:

$$
\begin{aligned}
\mathrm{r}_{\mathrm{t}}=\mathrm{M}\left\{\mathrm{y}_{\mathrm{t}} / \mathrm{m}_{\mathrm{i}, t}, \mathrm{D}_{\mathrm{i}, \mathrm{t}} ; \mathrm{i}=1,2, \ldots, k(k \leq 10\}\right. \\
\sum_{i=1}^{k} \int_{\mu_{i, t}} y_{i, t}\left(\mu_{i, t}, U_{i, t}\right) x P\left(\mu_{i, t} / m_{i, t}, D_{i, t}\right) d \mu_{i, t},
\end{aligned}
$$

where $P\left(\mu_{i, t} / m_{i, t}, D_{i, t}\right)$ - a priori to the time step $\mathrm{t}$ distribution of the probability density perturbations of $\mu_{\mathrm{i}, \mathrm{t}}$

For $y_{i}$, represented by expression (2), $r_{t}$ has the form:

$$
\mathrm{r}_{\mathrm{t}}=\mathrm{A} * \sum_{i=1}^{k} \exp \left(B m_{i, t}+B^{2} D_{i, t} / 2+C U_{i, t}\right),
$$

where $\mathrm{C}<0$.

It is easy to see that $r_{t}$ represents the sum of curves similar to the curves presented in Fig. 5, only the "weight" of the perturbation of here has two members. With precise knowledge of $\mu_{\mathrm{i}, t}$, its value is represented in Eq. (24) by means of M. E. of $m_{i, t}\left(D_{i, t}=0\right)$. When there is false knowledge of the disturbances $\mu_{\mathrm{i}, \mathrm{t}}\left(\mathrm{D}_{\mathrm{i}, \mathrm{t}}\right)$ 0 ), for its compensation it must be spent additional control of the $\mu_{\mathrm{i}, \mathrm{t}}$, which should be taken from the shared resource of phlegmforming hydrogen on the 1st isotope separation stage. And this could only be done at the expense of resource redistribution between units of this stage.

Minimum of $r_{t}$ risk was achieved by the programs of subsystem 3 of ACSTP of GPTP through a series of iterations, for each of which resource has been reallocated between the separating units with the highest and lowest values of members in the sum (24). Such algorithm of optimal control calculations was selected based on separability of the objective function $r_{t}$ and the convexity of its functions $\exp (\ldots)$ under the sign of the sum.

More delicate questions of adjustment of controls, derived from the condition of minimizing the conditional specific risk of $r_{s}$ for each step of the work of the ACS, with the aim of achieving the minimum value of the criterion $\rho_{\mathrm{s}}(6)$, taking into account the dual nature of the controls $\mu_{\mathrm{i}, \mathrm{s}}$, are not considered here.

\section{Results and Discussion}

\subsection{Tests Program of Adaptive Control System}

Development of ACS was completed in May 1975. By early August of that year, all programs of subsystems 1-3 of ACSTP were "hardwired" in ROM, and by the end of that month the Program of ACS tests was developed and agreed with the DDCP (and, in the first place, GPTP).

By the time of the ACS tests deuterium content in the worked off gas was reduced at GPTP to $2.2 \gamma$, and the degree of extraction increased to $94.9 \%$ from the 
project. Recall that at the beginning of the development of the ACS, these figures were $2.3 \gamma$ and $93.7 \%$, or in other words, over five years the output of heavy water was increased by $1.2 \%$ though the annual commitments of the GPTP collective were to increase it only to $0.1 \%$.

Under the Program, the tests of ACS were performed from 01.09.1975 to 31.10.1975. With that:

- tests were performed at constant loads for share gas in which the concentration of deuterium in the exhaust could be an indicator of the efficiency of the entire production of heavy water;

- during the first ten days of tests controls will not be issued and only statistical processing of data on all shifts must be carried out.

\subsection{Results}

The results of the tests of the ACS are shown in Fig. 6.

On the chart for August-October 1975, daily (averaged over shifts) the deuterium concentrations in the exhaust stream of hydrogen which was leaving GPTP are plotted.

As the graph shows, the daily concentrations in August were in the range from $1.2 \gamma$ to $3.2 \gamma$ with the average value of $2.2 \gamma$. The same character, this schedule carried into the first 10 days of September.

Starting from 1st shift of 11 of September at all 10 units flow rates of phlegmforming hydrogen were set according to calculations by subsystem 3 of ACSTP. For 3 days (9 shifts) the concentration decreased to the project, or in other words, to $1.4 \gamma$. And that process proceeded until October 10. And then in the next 5 days his character has changed. Why?

On the instructions of the supervisor of tests from the GPTP, the loads on the separating units, contrary to the requirements of the Program of ACS tests, were increased. The reaction of ACS on that situation is visible from the graph. After the transition process within 2 days the technological process at all of pre-concentration stage reached the purposive value of
1.6 $\gamma$. Though, the actual output of the target product of GPTP increased but this value fell according to the Program methodology of calculations focused on stable loads.

The loads were prohibitive for the production schedules and the supervisor had to refuse from them in 5 days. On the chart a small transition was again, and again deuterium concentration decreased to $1.4 \gamma$.

When to complete tests was a week, the concentration of deuterium in the hydrogen exhaust flow jumped under 3.0 $\gamma$ ! No one at first could not understand why. But when the "culprits" were found, they were the operators of the three most distant (from the authorities) units of the 8th, 9th and 10th. By agreement among themselves, in work logs, they wrote the assignments generated in the CCM subsystem 3, but the wheels associated with the needle valves, control costs phlegmforming hydrogen in units of separation, one of them for 3 days did not fit (Note that AAS level of liquid hydrogen in a cube of DC was mounted at the time, only on DC A3 aggregate and tested).

This "failure" in the test Program once again showed how the entire degree pre-concentration of deuterium is sensitive to the "distortions" in the process control throughout the stage and how effectively eliminates such distortions developed an adaptive ACS.

Results of the tests on the actual production of heavy water at the GPTP for the two months rose by $2.67 \%$. The fact that this rise is not increased to $5.1 \%$, there were a number of reasons, the main of which are clear to the reader from the above. But probably there were other unaccounted secondary factors, which somewhat underestimated the result.

The leadership of DDCP a roadmap for GPTP for next year was increased by $0.67 \%$. A larger percentage was left as a guide to what needs to come the employees of GPTP in the development of the established system. 


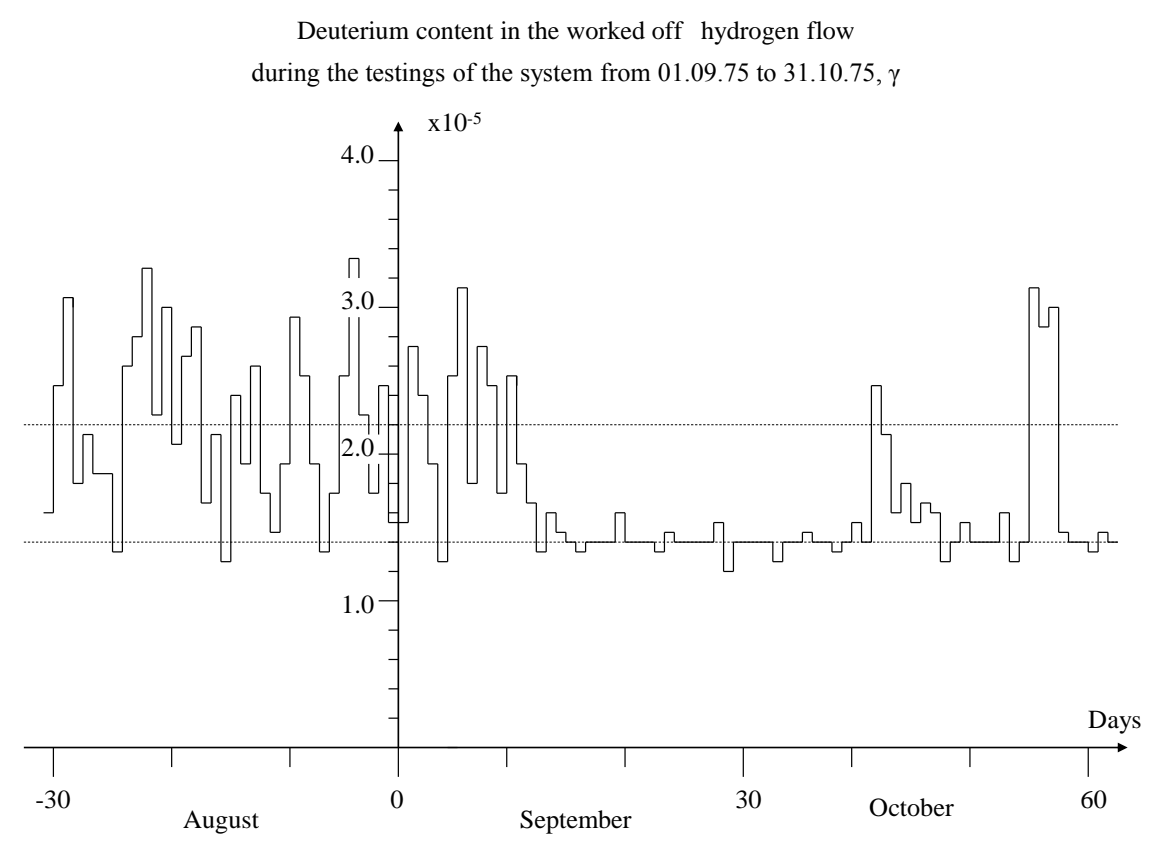

Fig. 6 The tests results of adaptive control system for the deuterium pre-concentration stage on GPTP.

\section{Conclusions}

The approach to the creation described here adaptive ACS would, in the opinion of the author, be used not only for control systems of modern production facilities of heavy water, but also find application in other technologies. For example, it could be used in a control system of compressor stations on gas pipelines.

\section{Acknowledgments}

I thank my colleagues who were part of the team I had the honor to lead. Eternal memory to those of them who left this light: Kolesnik Yury, Kozmin Petr, Massalsky Henry, Melnikova Zoya, Tkachenko Leonid. Long years to those who are alive today: Andreev Nikolay, Pozdnyakov Evgeny. I do not know the fate of those with whom we parted long ago: Victor Moroz, Alexander Khomenko.

I am grateful to my research supervisor Viktor Ivanenko, who helped me when working on my $\mathrm{PhD}$ thesis on the topic "Synthesis of an Automatic Optimization System for a De-benzene Unit (in
Ethylbenzene Production)" to acquire the necessary knowledge to create the control system described here in the future.

\section{References}

[1] Feldbaum, A. A. 1966. Fundamentals of Theory of Optimal Automatic Systems. Feldbaum.-M.: Publishing House "Science".

[2] Andreev, B. M. 1987. Heavy Isotopes of Hydrogen in Nuclear Engineering, edited by B. M. Andreev, Y. D. Zelvensky, S. G. Katalnikov. Moscow-Publishing House "Energoatomizdat".

[3] Malkov M. P. 1961. The Release of Deuterium from Hydrogen by the Method of Deep Cooling, edited by M. P. Malkov, A. G. Zeldovich, A. B. Fradkov, and I. B. Danilov, Publishing House "Gosatomizdat".

[4] Karachenets, D. V. The Control Unit of the Installation of Low-Temperature Rectification Process, edited by D. V. Karachenets, E. P. Pozdnyakov, L. N. Tkachenko, Y. A. Redin, Y. S. Rudoy, V. A. Ryabchiy, Ed.communication No. 9808.

[5] Ivanenko, V. I. 1969. The Tasks of Statistical Synthesis of Automatic Optimization Systems of the Massexchanging Installations, edited by V. I. Ivanenko, D. V. Karachenets and presented at the Fourth Congress of the International Federation of Automatic Control, Section 53, Warszawa. 